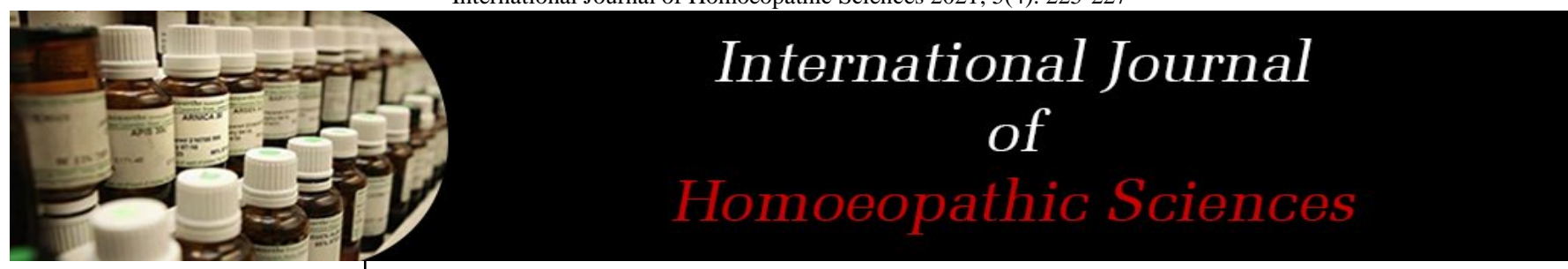

E-ISSN: $2616-4493$

P-ISSN: 2616-4485

www.homoeopathicjournal.com IJHS 2021; 5(4): 225-227

Received: 05-08-2021

Accepted: 02-10-2021

Dr. Vanita Rajiv Johari Professor HOD, Department of Materia Medica, Smt. CMP Homeopathic Medical College Vile Parle (West), Mumbai,

Maharashtra, India
Corresponding Author Dr. Vanita Rajiv Johari Professor HOD, Department of Materia Medica, Smt. CMP Homeopathic Medical College Vile Parle (West), Mumbai, Maharashtra, India

\section{How to use different ammunitions from the vast armamentarium of Materia Medica}

\author{
Dr. Vanita Rajiv Johari \\ DOI: https://doi.org/10.33545/26164485.2021.v5.i4c.475
}

\section{Abstract}

The vast data available for each remedy in Materia Medica has provided gigantic knowledge for their use. The application of Materia Medica is multi-dimensional and needs deeper insight and understanding for utility in day to day practice.

Keywords: tools of Materia medica, specifics, prophylaxis, constitutional, key-notes, totality, repertorization, suppressions.

\section{Introduction}

Different systems of medicines based on drug therapies have their own Materia Medica which gives direct indications to their uses in practice. Pharmacology of Modern Medicine gives physico chemical properties of the drug substances, while Dravya Guna (Ayurveda) describes Thridosha sidhanda (Vatha, Pitha \& Kapha) for understanding the indications of the bhasmas and kadas used for treatment.

Different ammunitions available in our enormous armamentarium of Materia Medica can find their application in clinical use in varied ways. Here are some examples of how to effectively use diverse tools hidden in our Armamentarium of Materia Medica.

\section{Application of different tools of materia medica}

1. Specifics: The proving of Cinchona bark brought out symptoms of Fever (auge) and Belladonna scarlet fever. Knowledge of Pathogenesis of the remedies can help one find direct indication for the drugs in a disease conditions. Homoeopathic specific remedies can be given on common symptoms of the diseases. Dr John Henry Clarke describes in his "Clinical Repertory" the use of the nosological correspondence, and Dr Oscar Boericke has added a therapeutic index in Boericke’s Pocket Manual giving Clinical Homoeopathic specific remedies for various ailments. Examples of specifics: Adonis vernalis - cardiac dropsy, Amyl Nitrosum - Angina Pectoris, Cholestrinum- Opacities of cornea, Erigeron- Placenta previa, etc., these remedies are usually prescribed in lower potencies as specifics.

2. Organopathic Indications: According to Dr J Burnett, drugs affect certain parts of the economy specifically; the general fact was elaborated originally by Paracelsus and then Hahnemann. Drs John H Clarke, R T Cooper, C M Boger and J C Burnett have given in their writings the experiences with respect to importance of selection of Organopathic remedies and their usefulness when other guiding symptoms are wanting. The so called rare and lesser known remedies also find their indications as organ remedies. As a part of Medicine / Surgery and Gynecology Therapeutics, these remedies are grouped as CNS drugs / Heart remedies/ Rheumatic drugs or Skin drugs, etc., For example, Physostigma for CNS disorders, Ceanothus for Spleen affections.

3. Prophylaxis and Genus Epidemicus: During an outbreak of an epidemic, we can make use of what is called a Genus epidemicus. This refers to a remedy or a group of remedies based on the totality of symptoms complex presented in most of the patients suffering from the same infection. The indicated remedy can also help as a prophylactic drug for individuals who have not brought out disease symptoms, but may have been exposed to infection. Aconite, Arsenic and Pulsatila are listed in Boericke's Repertory as Prophylaxis for Measles. A deep acting anti miasmatic remedy may also help in such cases. 
4. Ailments from - Immediate and Remote: Complaints may arise due to Physical or Mental cause. Hahnemann may be given the credit for identifying and giving importance to the psychosomatic connection for various diseases. Vulnerary remedies with their specific type of injuries help us in treating corresponding traumas. Preoperative and post-operative states too are seen in different remedies which when used judiciously will definitely help the patient. Effects of weather, food items, medicines, and abuse of narcotics are again seen differently in most of the remedies and can hence eradicate them. At the mental level long term results of anger, vexation and insults and mortification can be corrected with the help of the indicated drugs. Cause could even be recent or old.

5. Constitutional/Personalities type: Dr. James Tyler Kent was of opinion that it is the sick individual as a whole to be cured rather than the totality of characteristic symptoms of the diseased organs of the patient. Each human being makes his own disease, better said, he forms a pathology both of his psychic personality and of his physical organisms in accordance to deranged vital force. So often physical and mental temperament of the patient may help physician to decide the, for example, light eyes and light hair of Bromium, and cranky howling temper tantrums of Chamomilla. Dr Grovgyl had given an outline of constitutional disorders in the form of Hydrogenoid, Oxygenoid and Carbonitrogenoid dispositions. Remedies for the specific constitutional types can be considered once the physical and mental traits are traced.

6. General Characteristics or Totality: The art of Homoeopathy includes the ability to match a disease picture with a drug picture, similar to symptoms displayed in healthy person while proving the drug. Hahnemann taught that it was not necessary to find similarity so exact that one picture could be superimposed on the other, as readily as one can superimpose two triangles. The action is to be primarily in the mental sphere, then to a lesser extent the similarity has to obtain in the general bodily reaction and finally to even lesser extent to reactions of different systems and matched to drug pathogenesis and the natural disease. The search for drug among the plethora of the patient's symptoms can be likened to police search for an assailant, which is facilitated by an accurate 'identikit' picture and description, ignoring common features while concentrating on individual and distinctive descriptions.

7. Keynotes: In case of available PQRS symptoms in a given case we can easily find the corresponding similimum using the Keynotes given in Allen's Keynotes and Lippe's Redline symptoms. Key note can be a general modality like seen in Bryonia as aggravation from least motion and better by hard pressure and rest; or can be Red line mental state as described in word "TENSION" in Aconite.

8. Three legged totality (Stool): At times the data available in the case is too limited, the prescription in such a case can be made using the totality of the limited symptoms to get optimum results. It is generally believed that homoeopaths from Calcutta are very sharp at making their prescriptions on such totality and making good use of Boger's Synoptic keys and Allen’s Keynotes for their prescriptions.

9. Repertorization: On other occasions when there are too many symptoms and the picture doesn't seem to be clear. Maze of such symptoms can be easily repertorized and the result will help narrow down the list. Different types of repertories help in coming closer to the similimum. Dr Hahnemann had published a partial repertory in Latin. Use of Repertorization promotes discovery of a remedy which one had not thought of, or of a remedy one knows nothing of. Once the list of most probable drugs is available, the final selection can be made by referring back to source book or proving.

10. Dispositions and Lack of Reaction: All those qualities that differentiate a person from other person depend upon the exclusive nature of the vital dynamis which moves the body, all its systems, organs, tissues, cells of the body referred to as material organism by $\mathrm{Dr}$ Hahnemann. He was the first physician to ask, "What is to be cured?" The answer to which was given in his Organon as "It is the deranged vital dynamis to be cured.” We also understand certain people at certain age of their life suffer from ailments and diseases which are peculiar and uncommon and cannot be explained logically. Dr Hahnemann, Dr. J.H. Clarke and other authors have descriptions of these dispositions in their Materia Medica and repertories. For example during infant life the disposition of crying should be observed keeping in mind symptoms of Bell, Jalapa, Lyco, Pulsatilla and Psorinum. Besides dispositions, we also come across some cases where the sick individuals suffer from low vital dynamis on account of which we do not get symptoms which clearly point out the similimum. Dr Kent has given a list of remedies which belong to the rubric "Lack of reaction" in his Repertory. For example, Psorinum is indicated for lack of reaction in chilly, psoric persons who have despair of recovery.

11. Prescribing a Nosodes based on anamnesis: A Nosode is given in potentised form on symptomatic as well as clinical indications. Understanding of the usage of nosodes whether as constitutional medicine or specific or anti miasmatic has been beautifully described in various Materia Medica especially by Allens. Leads for choosing a nosode is often based on the medical history or in other words anamnesis.

12. History of Suppressions: Hahnemann has emphasized on removal of the cause disease for procuring cure in patients. Besides the immediate and remote causes, we have various factors that suppress the vital force leading to diseases. According to our science, there are three disease producing forces namely Psora, Sycosis and Syphilis. Practically all remedies have complaints arising from suppression of either physiological or pathological discharge or condition. 
13. Relationship of Remedies: Study of remedy Relation is most practical aspect of Materia Medica. The information provided can be applied directly for complimenting the previous prescription with deeper acting Anti-miasmatic remedy or using the chart to select an Acute remedy which follows well in case of an acute condition.

14. Mental Portrait: This appears to be the most favourite and challenging at the same time as it involves understanding the patients after careful case taking, analysis and evaluation and findings its closest reflection in Drug Picture. The toughest part is the more one tries to read and understand from different books, the more confusing it gets.

\section{Conclusion}

Application of above in clinical practice is quite enriching, only problem is that it does not come easy. Unfortunate part is lack of standardization. From understanding the language to get to the gist of the symptoms to breaking the prejudice of fixed images of the remedies in our minds, one has to be broadminded in our outlook to be able to use different methods/ tools for successful practice.

"The most expert healer is the man who knows how to handle his Materia Medica”, rightly said by C.M. Boger. Information or data available in Materia Medica texts are like words in a dictionary, one can learns how to use these words for communication only by mastering the language.

\section{Acknowledgment}

Express my heartfelt gratitude towards my colleagues, staff and patients at Smt. CMPH Medical College and Shree Mumbadevi Homeopathic Hospital, Mumbai for providing me with opportunity to apply theoretical knowledge in clinical practice.

\section{References}

1. Principles of Prescribing: Collected from Clinical Experiences of Pioneers of Homoeopathy: 1 By K.N. Mathur, B Jain Publisher, New Delhi, First Edition 2008.

2. Just You Listen: Dr Sarkar, Role of Materia Medica. Homoeopathic Medical Publishers. January 2019

3. Just You See: Dr Surinam Sarkar. Homoeopathic Medical Publishers, 2013.

4. Principles and Practice of Homeopathy Dr M L Dhawale, B Jain Publishers, Fourth Edition 2014. 